\title{
Addenda to the Program of the 33rd Annual Meeting of the Psychonomic Society St. Louis, Missouri \\ November 13-15, 1992
}

3-D/Movement Perception I

Touch

Categorization

Behavioral Pharmacology

Poster Session I

Vision

Judgment/Decision Making II

Cognition I
Abstract 53:

There was a third author on the paper: THOMAS F. SHIPLEY, University of Georgia.

Abstract 88: The third author's name is CATHERINE L. REED (rather than Catharine Reed).

Abstract 106: Withdrawn.

Abstract 115: Withdrawn

Abstract 156: Catherine L. Reed is now at Carnegie-Mellon University.

Abstract 189: Withdrawn.

Abstract 499: Withdrawn.

Abstract 505: There were two additional authors on this paper: DENIS J. HILTON, ESSEC, and BRIAN WALLACE, University of Wales.

The abstract's second sentence should read " "... lowest when they entertained two strongly competing hypotheses.",

Abstract 514: Withdrawn.

Abstract 518: $\quad$ Not given. 